\title{
A Review of the Use of the Asymptotic Framework for Quantification of Fretting Fatigue
}

\author{
D.A. Hills ${ }^{1}$ and D. Dini ${ }^{2}$ \\ ${ }^{1}$ Department of Engineering Science, Parks Road, Oxford OX1 3PJ, UK \\ ${ }^{2}$ Department of Mechanical Engineering, Imperial College London, London SW7 2AZ, UK
}

\begin{abstract}
We review the representation of fretting fatigue crack nucleation sites by simple asymptotic forms, and show that the multipliers on these fields produce very satisfactory quantifiers of the tendency of any contact (but particularly incomplete ones) to nucleate a crack. A procedure is suggested to adopt simplified contact geometries, easily tested under carefully controlled conditions in a laboratory, to assess the likelihood of the avoidance of all failure or to estimate the time needed for crack initiation. The results found are true material properties which may then easily be used to solve a wide range of practical problems.
\end{abstract}

\section{Introduction}

The nucleation of cracks from contact-edge sites, in the presence, usually, of differential slip, remains an incompletely understood phenomenon. When once a crack has started, early growth rate is controlled by a combination of remote (bulk) and contact stress fields, and that is properly quantified by classical fracture mechanics/Paris type equation approaches, but the conditions encapsulating nucleation are not. There are already several possible ways around this problem - the use of critical plane methods [1,2] or short crack growth ideas [3-5] being particularly attractive, whilst others have sought an analogy with the effect of notch on localising the stress field $[6,7]$. All of these methods have devotees and strong points but equally they have their weaknesses: critical plane methods assume that it is a combination of stress components which are responsible for controlling nucleation, and, in their original form, there is no concept of size effects. Short crack growth methods are attractive because they can be followed with little more than Paris data (plus the bulk stress fatigue limit) to hand, but they lack a real physical basis and are, in any case, really concerned with the early development of an existing (if short) crack. Equally, notch analogies are just that - there is some matching of stress gradients between the fretting problem and some 'equivalent' notch, but it certainly not possible to match up the whole of the local stress field between the two problems.

The idea advanced here is rather different. There is no suggestion that we need to understand the mechanics of the nucleation process (which is, in any case, likely to vary from material to material and governed by microstructural features), but rather that we carry out carefully controlled laboratory tests which reveal material properties, viz. the multiplier on the local stress field (an asymptotic form) in the neighbourhood of the contact edge, and we use that both as a means of finding the threshold for nucleation and, at higher loads, the number of loading cycles needed to start a crack. We would like this method to be comprehensive, in the sense that it could be applied to any kind of contact, and so, before we explain the method further, we look at the possible kinds of contact which may arise and their characteristics.

Figure 1 shows schematically the main kinds of contact found and, in this discussion we will assume that both elements are made from the same material. Figure $1(a)$ is an incomplete contact, where at least one of the bodies is convex, and where the application of an increasing normal force causes 
the contact to get bigger. Hertzian contacts fall into this category, and an important characteristic is that, if we measure the contact pressure, $p(s)$, in the neighbourhood of the contact edge it is always of the form $p(s)=K_{N} \sqrt{s}$, where $s$ is a coordinate measured inwards from the edge and $K_{N}$ is a measure of the strength of the square root-bounded field. A consequence of this is that if remote loading (the application of a shear force or differential remote tension) is such as to generate shear tractions, there will, with rare exceptions, always be a slip region, producing fretting damage, attached to the contact edge. Figure $1(\mathrm{~b})$ is a complete contact (or conformal in Barber's notation [8], because the profiles of the unloaded bodies conform), and where the size of the contact is independent of the contact force, but is defined, instead, by an abrupt change in profile ('corner'). Problems of this class are considerably more complicated, and there are several possible responses, but the best way, usually, to look at their behaviour is to fit a Williams semi-infinite notch solution into a contact edge region including both bodies, that is, we initially assume that the contact edge is locally adhered. From this we can immediately infer the coefficient of friction needed to maintain that adhesion and therefore decide whether, in any particular case, the contact edge suffers fretting or merely remains adhered, when the assembly simply looks like a monolithic notch. The implied local stress state at the contact edge is of the form $p(s)=K_{I} s^{\lambda-1}$, where $\lambda$ is an eigenvalue of the problem, and the local state of stress is singular, with $K_{I}$ being a measure of the intensity of the field in the neighbourhood of the contact corner. There are circumstances when the contact edge separates, but when this does occur the contact them falls into the incomplete category described earlier. More details are provided in recent publications aimed at unravelling the mechanics governing fretting in complete contacts [9-15].

Figure 1(c) shows a contact which, upon the application of an infinitesimal normal load recedes, that is, it snaps to a smaller size than that originally present. Contacts of this class are still being studied to understand more of their properties. When once the contact has receded it has many of the same properties as an incomplete contact. Lastly, Figure 1(d) shows a contact formed from two bodies of the same size where there are no necessary local stress gradients. Properties of this kind of contact are not well understood and we exclude them from the remainder of the discussion.

\section{Development of asymptotic solutions}

It will be appreciated that the two main forms of contact edge behaviour arising are square root bounded, in the case of incomplete contacts and power order singular in the case of complete contacts. The way we need to treat them is wholly different, and we will consider the incomplete contact edge first.

We retain the assumption that the contacting bodies are made from the same material and, whatever the conditions are remote from the contact edge, there will always be some scale at which the local solution will look like that formed between two half-planes, Figure 2. In many cases the half-plane idealisation will apply across the whole of the contact interface. Suppose that the normal load, $P$, forming the contact, is applied first and held constant, so that, in the subsequent loading, the location of the contact edge is fixed. No shear tractions will arise at this stage but imagine, for the time being, that the coefficient of friction between the bodies is infinitely high. If now other remote loading is applied such as an increasing shear force, $Q$, or a difference in bulk tensions, $\sigma$, either side the interface, a local shear traction will develop which is square root singular in form, i.e. we may write the local shear traction, $q(s)$, as $q(s)=K_{T} / \sqrt{s}$. In complicated problems the connexion between the remote loading and these local generalised stress 'intensity' factors (in the case of the contact pressure there is, of course, no intensification) will have to be found by numerical methods, but for simple problems such as a Hertzian contact or a 'flat and rounded' contact closed form calibrations 
may be found, and some examples are given in Refs. [16-17]. In the neighbourhood of the contact edge the local stress field (for the case when the coefficient of friction is infinitely high, inhibiting all slip) is completely defined by the two stress intensity factors $K_{N}, K_{T}$. Suppose, now, that we relax the assumption that the friction be infinitely high, and reduce it to a value, $f$. The contact pressure will certainly remain unchanged and, provided that the conditions are not so extreme as to permit a very long slip zone to develop, the shear traction will continue to be square root singular in form in the adhered hinterland. The slip zone length, $d$, is given by [17]: $d=\frac{K_{T}}{f K_{N}}$. So, cracks may nucleate at or very close to the contact edge, invariably within the slip region, and the whole of the local field - stress state, slip displacement is controlled by just these three quantities $\left(K_{N}, K_{T}, \mathrm{f}\right)$. It follows that any pair of contacts made with the same material, and experiencing the same history of $K_{N}, K_{T}$ ( $f$ will, of course, necessarily be the same if we take it to be a material constant) will have the same tendency to nucleate a crack. Thus, a laboratory experiment carefully conducted using a simple contact pad geometry and with given loading history may be expected to have exactly the same crack nucleation properties as a complicated prototype experiencing the same history, as encapsulated by $K_{N}, K_{T}$. We will return to this point later.

We turn, now, to the case of complete contacts, and complete contacts edges, Figure 3 . And, the first thing to note is that this is very different indeed from a half-plane formulation, and that the appropriate local domain to consider, as stated earlier, is a semi-infinite wedge of total internal angle $\pi+\varphi$, where $\varphi$ is the angle at the edge of the contact-defining body. Detailed consideration of the Williams solution provides valuable insight. Not only do we learn the orders of singularity of the dominant eigensolutions from the characteristic equations, but the eigenvectors enable us to find the traction ratio arising along the line of the contact. This gives us the minimum coefficient of friction needed to achieve adhesion in both mode I dominated and in mode II dominated (if it exists) regions. These results are provided in Ref. [14], and it will be noted that the coefficients of friction needed to ensure stick are comparable with the range of coefficients of friction experienced in practice. For example, for a contact edge angle of $\varphi=90^{\circ}$ the critical coefficient of friction is 0.54 . It follows that, in contacts where this coefficient of friction is exceeded the contact edges will never slip, unless the applied loading is such as to produce a positive local stress intensity, and therefore separation, at some point in the loading cycle so that formally the contact becomes incomplete. If intimate contact to the edges is always maintained only interior points may slip initially (and the last point to slip will be the contact edge). In these cases the slip region is fully contained, and the slip displacement therefore relatively small. Experience shows that fretting fatigue is not observed starting from an interior point of a contact. If the coefficient of friction is insufficient to maintain adhesion slip zones will occur at the contact edge initially, but shakedown will occur rendering the steady state slip zones very small indeed [10]. We conclude that fretting fatigue as usually understood is not a phenomenon which arises in the case of complete contacts. Of course fatigue failure may, indeed, start from the edge of a stationary contact of this class, but it is much more likely that this is really a 'notch fatigue' problem.

\section{Experimental Evidence}

\subsection{Incomplete Contacts}

Many fretting fatigue tests have been conducted over the years in several laboratories throughout the world, almost always using Hertzian contacts in the well-controlled cases. A comprehensive comparison of the fretting fatigue strengths measured, but using the asymptotic principles described above, was published [18]. Here, we will provide a summary in the form of one graph, Figure 4, 
which gives the fretting fatigue strength as a function of $\Delta K_{T}$ alone for aluminium alloys [19, 20]. A full description of the local state of stress would clearly need to make use of $K_{N}$, too, but this is constant in time and we therefore expect its value to have only a secondary effect on fatigue life. The 'S-N' curve, with a clear fatigue limit is gratifying, and even more so is the fact that the size effect Nowell observed [19] seems completely to be taken into account, whereas a comparison based on stress range alone omits this effect and no systematic correlation is found. The contact edge stress intensity factors control only the state of stress in the neighbourhood of the edge (typically up to about $10 \%$ of the contact width) so that they do not provide useful information about the propagation of cracks. They therefore do not give a full description of the total life of a fretting fatigue specimen, but only the nucleation 'life'. In many cases, when once a crack has formed and grown to a significant size the time (meaning number of loading cycles) needed to propagate it to failure may be a relatively small fraction of the total life.

\subsection{Complete Contacts}

The evidence available to back up the equivalent correlation in the case of complete contacts is less compelling. Many tests were conducted at Oxford fifteen years ago using complete contacts pressed onto dogbone specimens made from an ultra-high strength steel (super CMV), but those tests were conducted before the underlying theory was developed and they showed a great deal more scatter than we had expected [11]. This may partly because of experimental difficulties - it is extremely difficult to arrange for the contact shear force to lie exactly in the contact plane without a moment being present, and it was also not understood at the time that what the possible regions of interfacial slip might be. As stated earlier, the theoretical evidence is that fretting fatigue, in a conventional sense, ought never to be a problem with complete contacts, unless the stress intensity at the 'trailing' edge is sufficient high to cause local separation, when the contact becomes, of course, formally incomplete anyway. At lower values of stress intensity (not producing macroscopic opening) then either; (a) at high values of friction points within the interior of the contact slip but the edges remain stuck, but there is no evidence that cracks ever form from interior points or (b) at lower coefficients of friction most of the corner will shake down to an adhered state leaving only a minute region of cyclic slip, much less than that normally associated with fretting damage.

Recent work by Lehtovaara and others [21] has sought to measure fatigue life in a material where the geometry of the contact includes an interface terminating at a contact edge, using a clamped cantilever. Certainly fatigue life was measured, and whether fretting damage modified the 'notch' fatigue certainly experienced is not clear. The authors chose to display their results in terms of total life on a 'conventional' $S-N$ plot, Figure 5, but ignored the singularity and used for the value of ' $S$ ' (stress), the direct stress found at the root of the cantilever implied by elementary bending theory. Clearly, the results when displayed in this form could not be carried over to any other geometry. In a discussion document [22] we took the same experimental test results but collocated the Williams' notch solution into the cantilever root corners. Flexure of the cantilever tip of course excites both eigensolutions, but the assumption was made that the process zone was sufficiently small for it to be controlled by the mode I stress intensity factor. So, the S-N results may be re-plotted on a delta $\mathrm{KI}$ versus life graph, and this is shown in Figure 6.

Other experimental using the celebrated traditional 'bridge' geometry [23] cannot easily be re-worked into this form because the loads applied invariably caused local separation of the bridge-foot edges [24], so that, during the loading cycle the critical region experiences a complicated cycle during part of which the contact corner is closed and during another part it is open (and incomplete). The range of Mode I stress intensity could still be used as a measure of the local stress field but only (a) 
assuming that the most positive value was not so high as to cause the separation region to stray beyond the region in which the $K_{l}$ field dominated, and (b) it would seem likely that the fretting strength would be controlled by $\Delta K_{I}$ if $K_{l, \min }>0$, and by $K_{l, \max }$ if $K_{l, \min }<0$.

\subsection{Other types of Contacts}

There are no known experimental results on the fretting fatigue strength of either common edge or receding contacts. In the case of the former much more work to characterise the edge field needs to be done, and this may be quite difficult. In the case of receding contacts there is clearly quite a lot in common with incomplete contacts - the main extra problem is simply that the contact edge is not known. Also, depending on the load history, it may well be the case that the contact recedes during part of the load cycle but becomes complete during the remaining part.

\section{Discussion}

\subsection{Incomplete Contacts}

The method hinges on a 'one term' approximation to both the contact pressure distribution adjacent to the contact edge, and the nature of the shear traction distribution. It is important for both of these terms to be a good approximation to the contact tractions over a region which more than spans the slip zone, as we assume, when finding the slip zone size, that these are the two characteristics which define the 'inner' solution - the presence of slip. The contact pressure representation is a term from the eigenfunction expansion of a wedge of internal angle $\pi$ radians where one face is traction free whilst the other is subject to a constant displacement $u_{\theta}$. It follows that the contact pressure representation is equally suited to all incomplete profiles, although the length over which it is valid will vary greatly. One of the biggest limitations of the treatment of the slip solution, at the moment, is that it is assumed that the contact normal load remains constant so that not only is magnitude of the near-edge contact pressure constant, but also the location of the contact edge does not change. This is something we shall address in the future, although asymptotes are more difficult to apply when the normal load varies because they are attached to the contact edge, and its location varies with time.

It is, perhaps, worth remarking how the interaction between frictional slip and plastic slip enters the problem. We assume that the nucleation of a fatigue crack is ultimately due to irreversibilites in plastic deformation during cyclic loading. Cracks invariably start somewhere quite near the contact edge; usually not at the very edge but either within the slip region or at the stick slip interface. The whole of the procedure being defined relies on the strain history in this neighbourhood being characterised by the asymptotes. But, just as the arguments for fatigue crack propagation rely only on the plastic zone being well within the region where the crack tip stress intensity factors define the strains, the same applies here. Thus, in the case of strong materials the plastic zone may be confined to a region immediately beneath the slip zone but in a softer material it may encompass the whole of the slip zone. It will be noted that this does not invalidate the argument provided only that this lies, as stated above, within a region in which the one-term asymptotes apply. Other approximations are consequential on the use a single term asymptotes; in particular, the size of the slip zone is given only approximately by the formula stated.

\subsection{Complete Contacts}

In the case of complete contacts although, in theory, we could use a combinations of modes I and II Williams eigensolutions, and, indeed, we could also employ the slipping corner asymptote, in 
practice we will normally assume that it is the range of the mode I (dominant) eigensolution which controls the corner state of stress alone, at any rate for a sensible range of contact corner angles. The presence of the mode II component effectively defines an outer limit to this mode I dominated form (when the characteristic length do is approached), and the presence of slip, although it will modify the nature of the local tractions, does not, as above, detract from the strength of the asymptotic form as a correlator of nucleation life. The only occasion when this argument does break down is when the coefficient of friction is so low that the slip zone envelopes the mode I dominated region, and, when this arise the whole concept of using asymptotes is called into question as the 'outer' solution is no longer unique. But, for example, for a contact having an edge internal angle of $\pi / 2$ radians, the critical coefficient of friction to ensure adhesion is 0.54 , and, in practice, common alloys develop a coefficient of friction bigger than this, and hence the contact corner and counterface effectively become adhered to form a 'notch' - in fact, with a notch radius which is smaller than those normally attained when a sharp notch is machined. Under these circumstances we would expect a complete contact and geometrically similar notch in a component made from the same material, and experiencing the same range of stress intensity to have the same nucleation life, although this has yet to be tested experimentally.

\subsection{Fretting Fatigue Life Prediction and Experimental Corroboration}

A means of quantifying fatigue life has been proposed, based on the idea that the local stress field encompassing the process zone is controlled by one of a set of asymptotic fields. The nature of the field really depends on whether the contact being studied is 'complete' or 'incomplete'. There is a great deal of experimental evidence already available in the case of incomplete contacts (see e.g. [18] and references therein, including [19, 20, 25, 26]), and which point to the range of stress intensity for the shearing part of the load as being the key parameter. For any given material, two life plots are needed - one for the shear traction stress intensity appropriate for incomplete contacts, and one for the mode I Williams stress intensity - for complete contacts. The amount of data available for complete contacts is quite limited (see [11, 12, 21, 23]). However, given the strong similarities between complete contacts and sharp notches, it is envisaged that (more abundant) data obtained for notched specimens (see e.g. [27]) may also significantly contribute to shed light on the suitability of asymptotic approaches to capture fretting fatigue life of complete contacts.

One aspect of the interpretation of the results which has not been mentioned is that in most experiments we measure total life. When a crack has formed it propagates under the action of both the local contact stress field and any remote loads, but the concepts of fracture mechanics continue to apply, i.e. that with the usual Paris assumption, it is simply the range of mode I stress intensity (here defined at the crack tip) which controls the fatigue life. Plotting crack length evolution as a function of number of cycles reveals that the propagating crack normally evolves very quickly, and that the majority of the crack life is expended in nucleation and early development. But in plotting total life against the nucleation intensity factor we tacitly assume that the whole of the life is consumed in nucleation, and fail to subtract the propagation part of the life. The main reason for this is, of course, that it is very difficult to decide at what point the crack nucleation process stops and propagation takes over. It is conceivable that early propagation itself takes place within a region in which the state of stress is quantified by the nucleation 'intensity' factor.

\section{Conclusions}

The method described would seem to be quite viable as a practical procedure, enabling laboratory test to be matched to complex prototype, and that was one of its main aims. It also does not matter, 
in the case of complete contacts, whether any fretting damage is, in fact, incurred, and enables notch and sharp edged fretting fatigue to be treated uniformly.

Extensions to the concepts are under way to generalise these ideas further. For example, it should be possible to devise a lifing strategy which includes the possibility of the normal load also varying cyclically. A further extension is to combine the ideas described here with those used to incorporate small amounts of rounding into notionally complete contacts so that the full range of problems - not just those which are at the 'very sharp' or 'fully rounded' ends of the spectrum may be treated.

\section{References}

[1] Goh, C.-H., Wallace, J.M., Neu, R.W., and McDowell, D.L., Polycrystal plasticity simulations of fretting fatigue, International Journal of Fatigue, (2001), 23(1), 423-435.

[2] Carpinteri, A., Ronchei, C., Spagnoli, A., Vantadori, S., Lifetime estimation in the low/mediumcycle regime using the Carpinteri-Spagnoli multiaxial fatigue criterion. Theoretical and Applied Fracture Mechanics, (2014), 73, 120-127.

[3] Dini, D., Nowell, D., Dyson, I.N., The use of notch and short crack approaches to fretting fatigue threshold prediction: theory and experimental validation, Tribology international, 2006, 39(10), 11581165.

[4] Fouvry, S., Nowell, D., Kubiak, K., Hills D.A., Prediction of fretting crack propagation based on a short crack methodology, Engineering Fracture Mechanics, (2008), 75(6), 1605-1622.

[5] Araújo, J.A., Castro, F.C., A comparative analysis between multiaxial stress and $\Delta \mathrm{K}$-based short crack arrest models in fretting fatigue, Engineering Fracture Mechanics, (2012), 93, 34-47.

[6] Nowell, D., Dini, D., Stress gradient effects in fretting fatigue, Tribology International, (2003), 36(2), 71-78.

[7] Bohórquez, L., Domínguez J., Elastic-plastic analysis of a punch and its equivalent notch under fretting conditions, International Journal of Mechanical Sciences, (2013), 66, 120-131.

[8] Barber, J.R., Elasticity, Springer (Dordrecht) $3^{\text {rd }}$ edn., 2010.

[9] Churchman, C.M., Hills, D.A., General results for complete contacts subject to oscillatory shear, Journal of the Mechanics and Physics of Solids, (2006), 54(6), 1186-1205.

[10] Churchman, C.M., Hills, D.A., Slip zone length at the edge of a complete contact, International Journal of Solids and Structures, (2006), 43(7-8), 2037-2049.

[11] Hills, D.A., Paynter, R.J.H., Dini, D, An overview of the quantification of fretting fatigue lives of complete contacts, Engineering Fracture Mechanics, (2012), 80, 3-12.

[12] Flicek, R., Hills, D.A., Dini, D., Progress in the application of notch asymptotics to the understanding of complete contacts subject to fretting fatigue, Fatigue \& Fracture of Engineering Materials \& Structures, (2013), 36, 56-64.

[13] Flicek, R.C., Hills, D.A., Dini, D., Sharp edged contacts subject to fretting: A description of corner behaviour. International Journal of Fatigue, (2015), 71, 26-34. 
[14] Hills, D.A. and Dini, D. What level of friction guarantees adhesion in a complete contact?, Journal of Strain Analysis for Engineering Design, (2004), 39(5), 549-551.

[15] Flicek, R.C., Hills, D.A., Barber, J.R., Dini, D., Determination of the shakedown limit for large, discrete frictional system. European Journal of Mechanics - A/Solids, (2015), 49, 242-250.

[16] Dini, D., Hills, D.A., Bounded asymptotic solutions for incomplete contacts in partial slip, International Journal of Solids and Structures, (2004), 41(24), 7049-7062.

[17] Dini, D., Sackfield, A., Hills D.A., Comprehensive bounded asymptotic solutions for incomplete contacts in partial slip, Journal of the Mechanics and Physics of Solids, (2005), 53(2), 437-454.

[18] Hills, D.A., Thaitirarot, A., Barber, J.R., Dini, D., Correlation of fretting fatigue experimental results using an asymptotic approach, International Journal of Fatigue, 43, (2012), Pages 62-75.

[19] Nowell D., An analysis of fretting fatigue, D.Phil Thesis, University of Oxford; 1988.

[20] Szolwinski, M.P., Farris, T.N., Observation, analysis and prediction of fretting fatigue in 2024T351 aluminum alloy, Wear, (1998), 221(1), 24-36.

[21] Juoksukangas J., Lehtovaara A., Mäntylä A. Development of a complete contact fretting test device, Proceedings of the Institution of Mechanical Engineers Part $J$ - Journal of Engineering Tribology, (2013), 227, 570-578.

[22] Flicek, R.C., Hills, D.A., and Dini, D., A discussion of: Development of a complete contact fretting test device by J. Juoksukangas et al., Proceedings of the Institution of Mechanical Engineers Part $J$ - Journal of Engineering Tribology, (2014), 228(1), 123-126.

[23] Noraphaiphipaksa N, Kanchanomai C, Mutoh Y. Numerical and experimental investigations on fretting fatigue: relative slip, crack path, and fatigue life, Engineering Fracture Mechanics, (2013), 112, 58-71.

[24] Hills, D.A., Flicek, R.C., A discussion of "Numerical and experimental investigations on fretting fatigue: Relative slip, crack path, and fatigue life" by N. Noraphaiphipaksa, C. Kanchanomai, and Y. Mutoh., Engineering Fracture Mechanics, (2015), 133, 52-55.

[25] Lykins, C.D., Mall, S., Jain, V., An evaluation of parameters for predicting fretting fatigue crack initiation, International Journal of Fatigue, (2000), 22, 703-716.

[26] Murthy, H., Mseis, G., Farris, T.N., Life estimation of Ti-6Al-4 V specimens subjected to fretting fatigue and effect of surface treatments, Tribology International, (2009), 42(9), 1304-1315.

[27] Flicek, R.C., Hills, D.A., Dini, D., Refinements in the characterisation of mode-mixity and small scale yielding at sharp notch roots, Engineering Fracture Mechanics, (2014), 126, 73-86. 


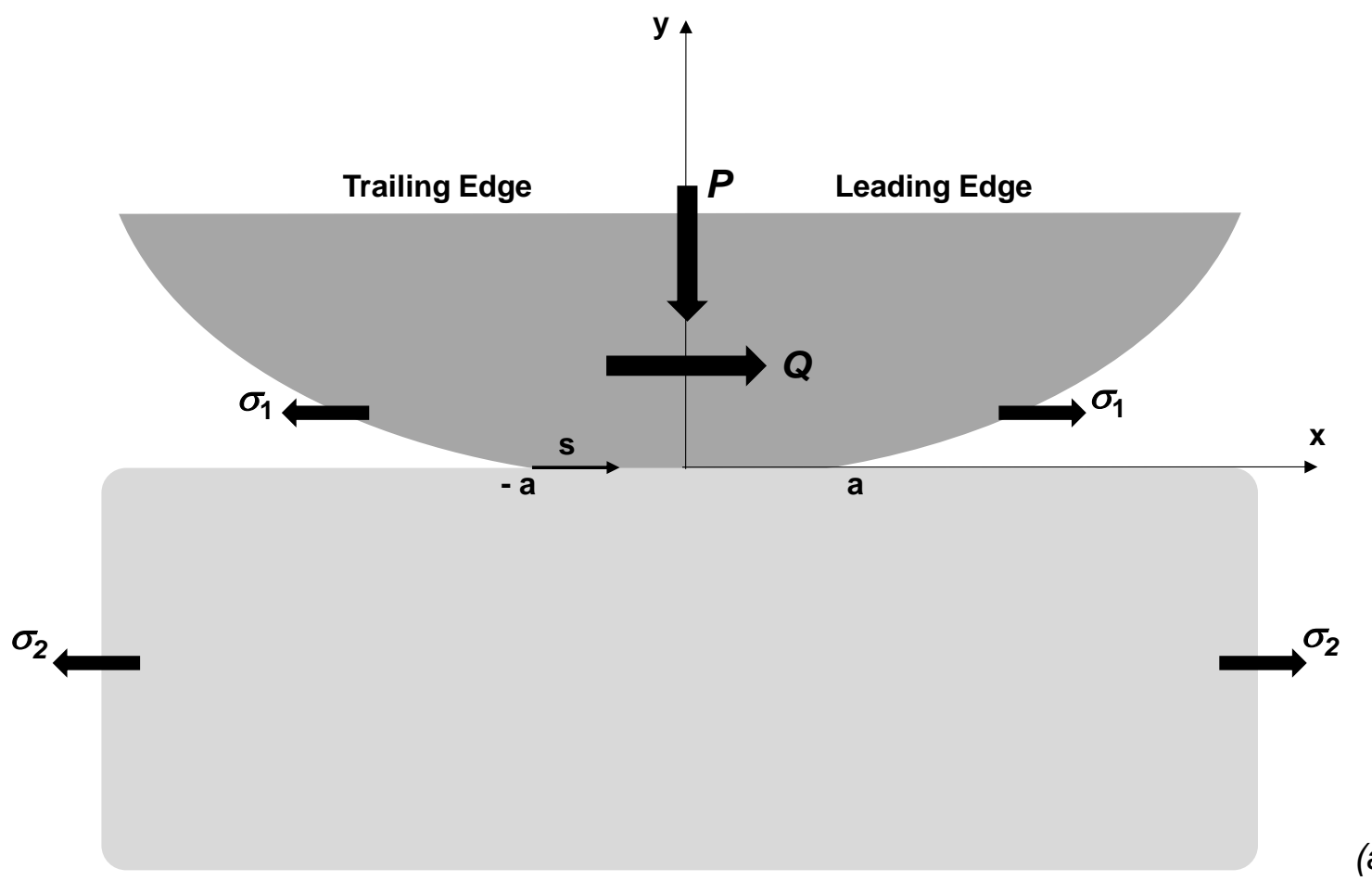

(a)

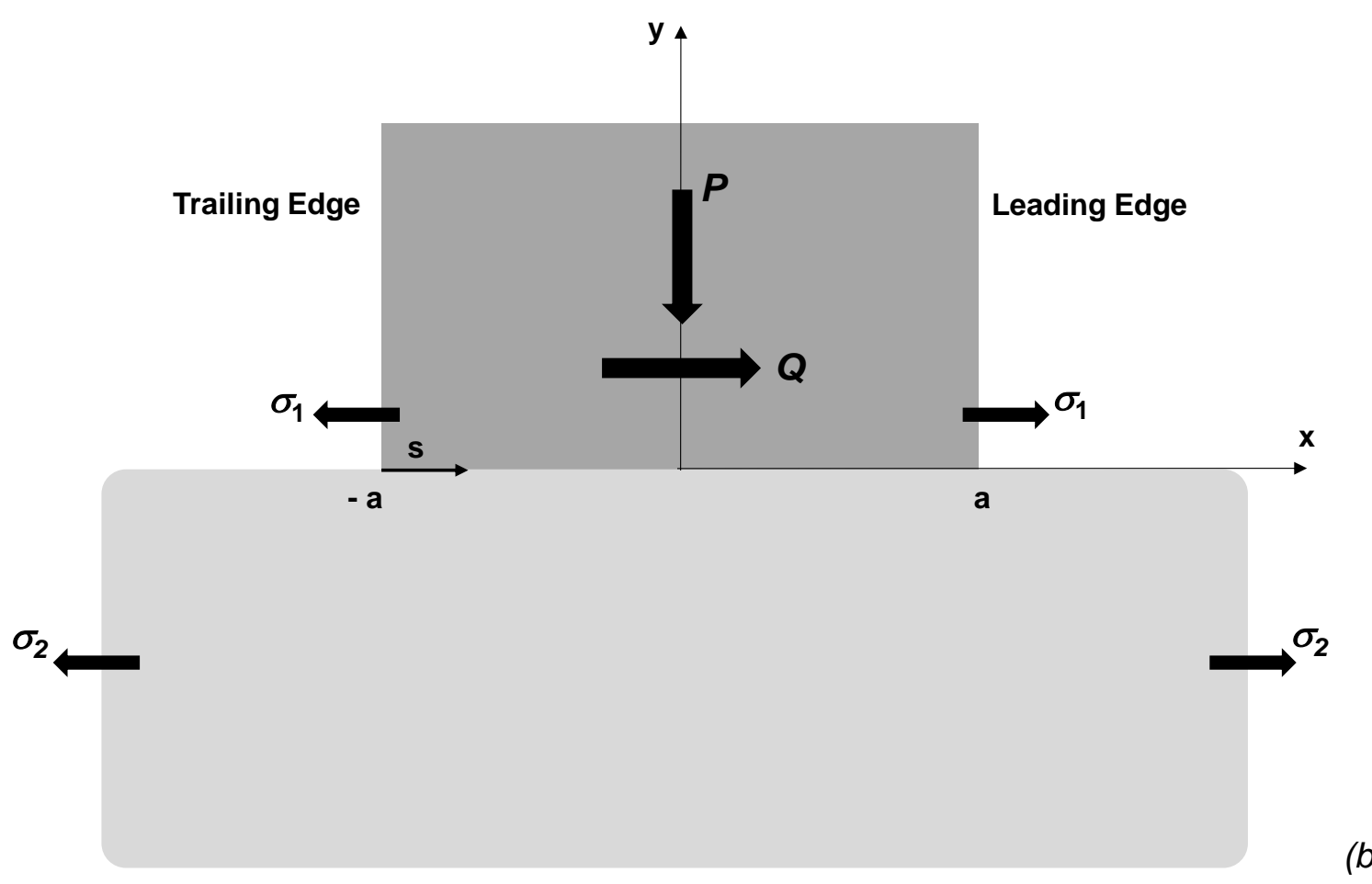

(b) 


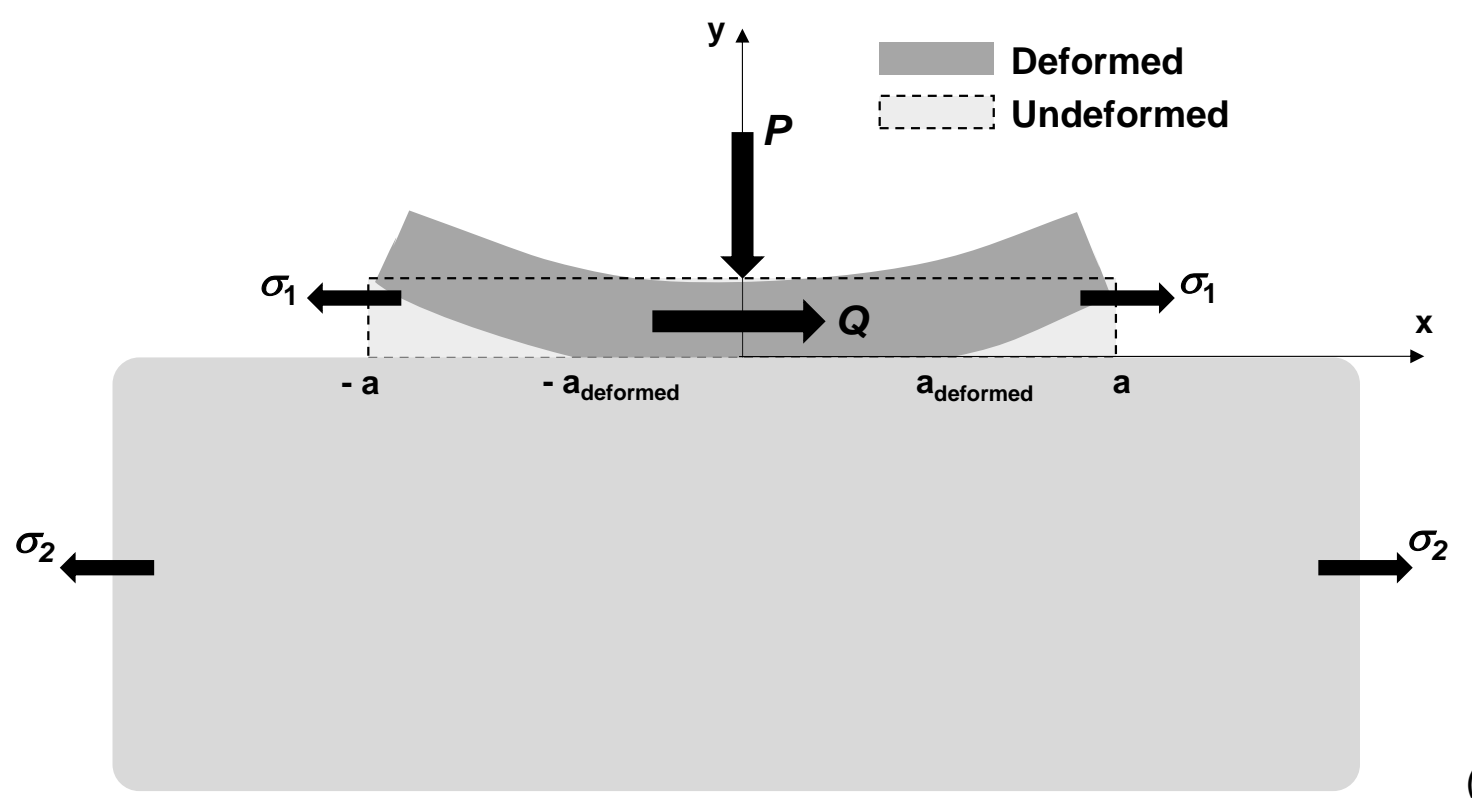

(c)

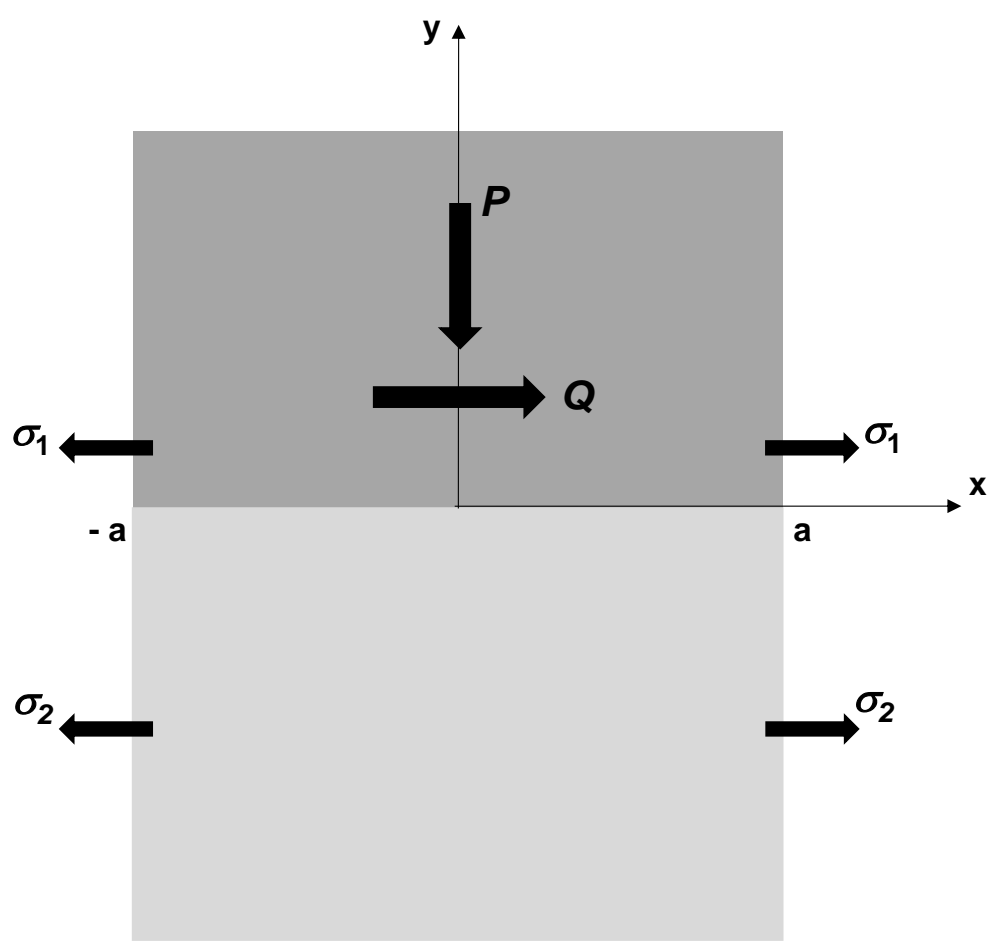

(d)

Figure 1. Schematic showing different classes of contact problems;(a) incomplete, as exemplified by the Hertzian geometry, (b) complete, exemplified by a square block pressed onto a half-plane, (c) receding, such as when a central force is applied to a strip resting on a half plane, and (d) common edge such as when two blocks of the same size are pressed together. In all cases the contacting elements are made from the same material. 


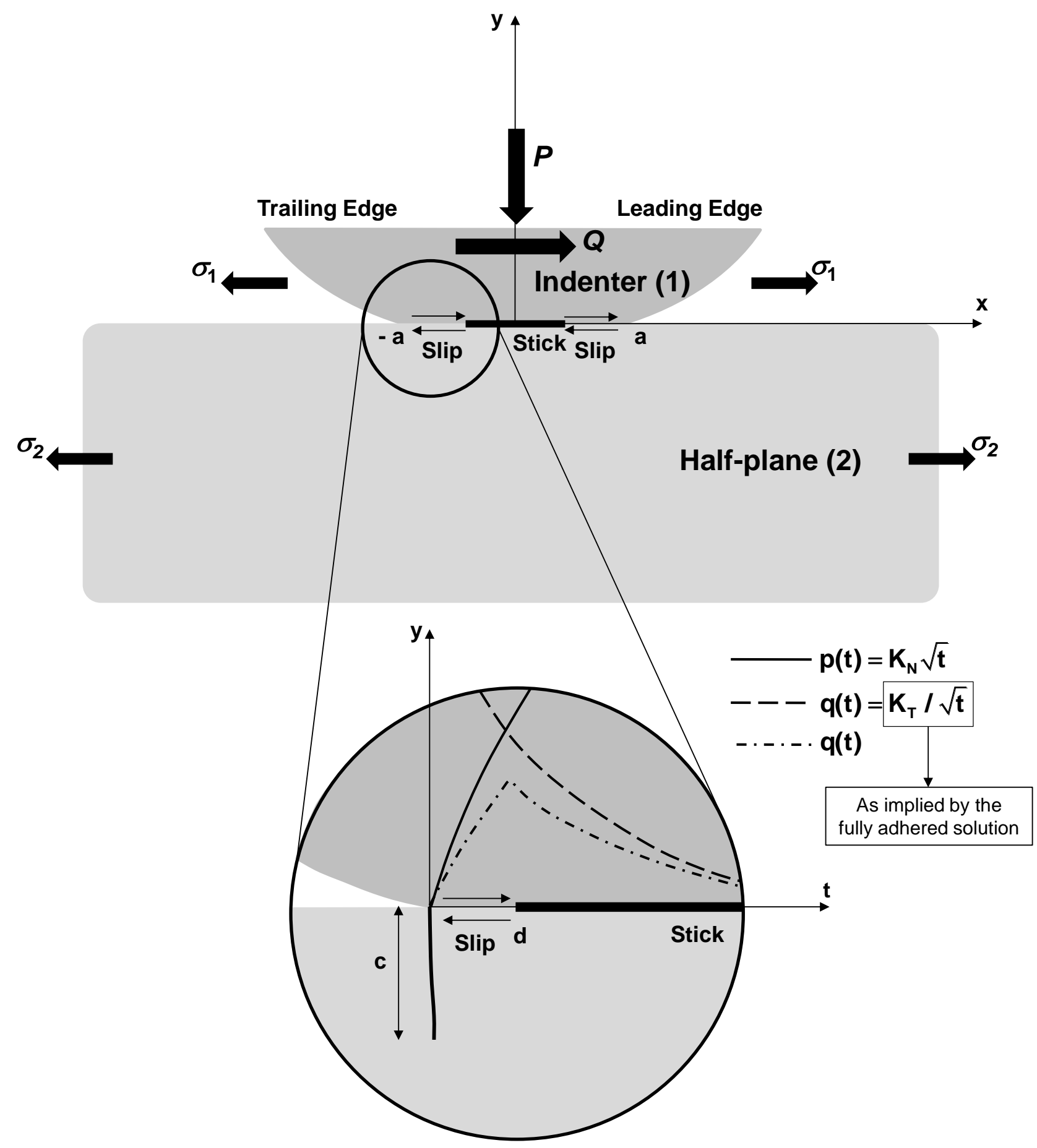

Figure 2.An incomplete (here Hertzian) contact subject to normal loading, shear and differential tension, and the representation of conditions at the contact edge by asymptotes $K_{N}, K_{T}$. 


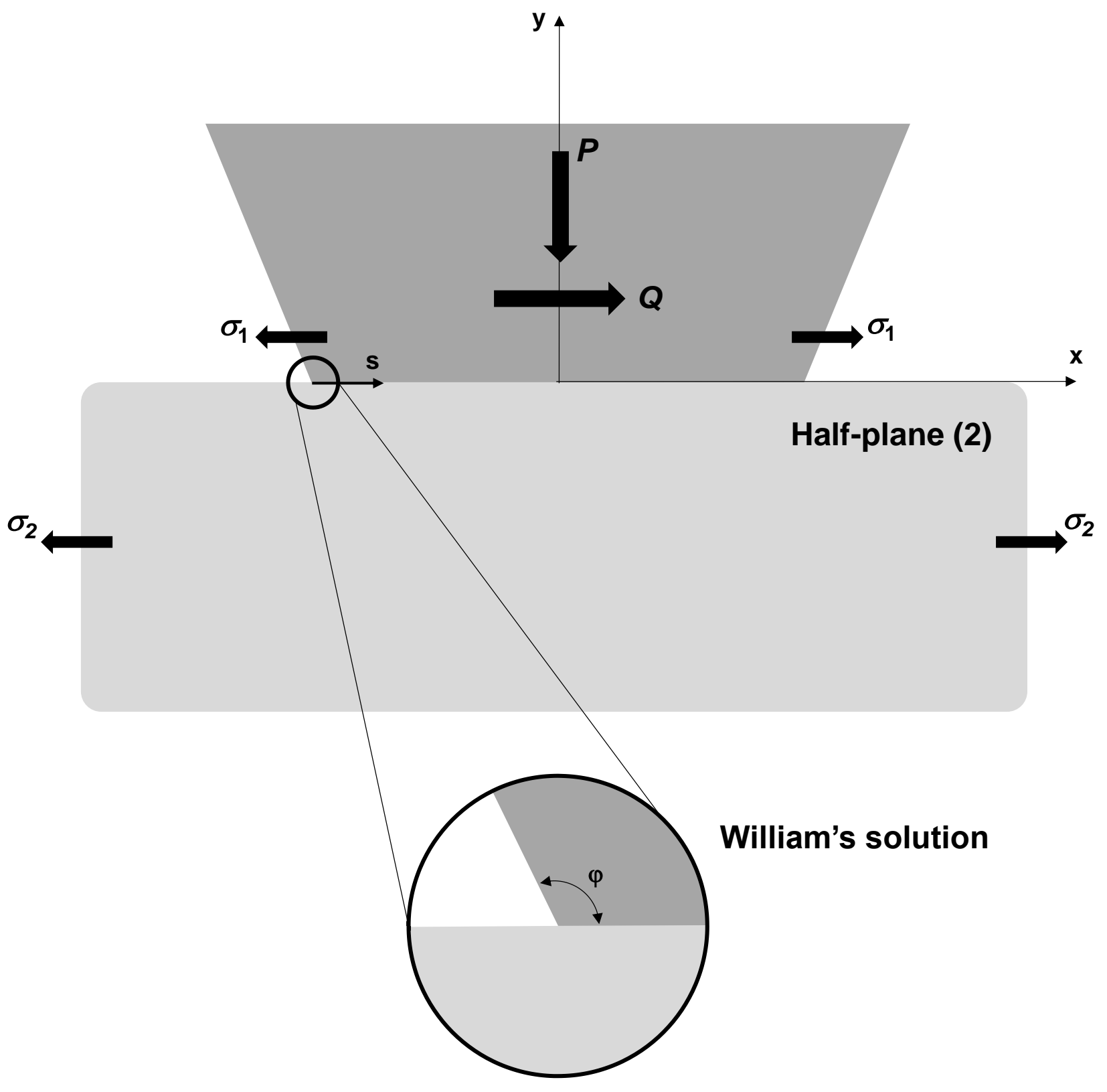

Figure 3. A complete contact, here a trapezium punch pressed onto a half-plane, and having adhered edges so that the contact tractions and indeed whole stress field adjacent to the contact edge may be represented by a wedge of total internal angle $\pi+\varphi$ radians. 


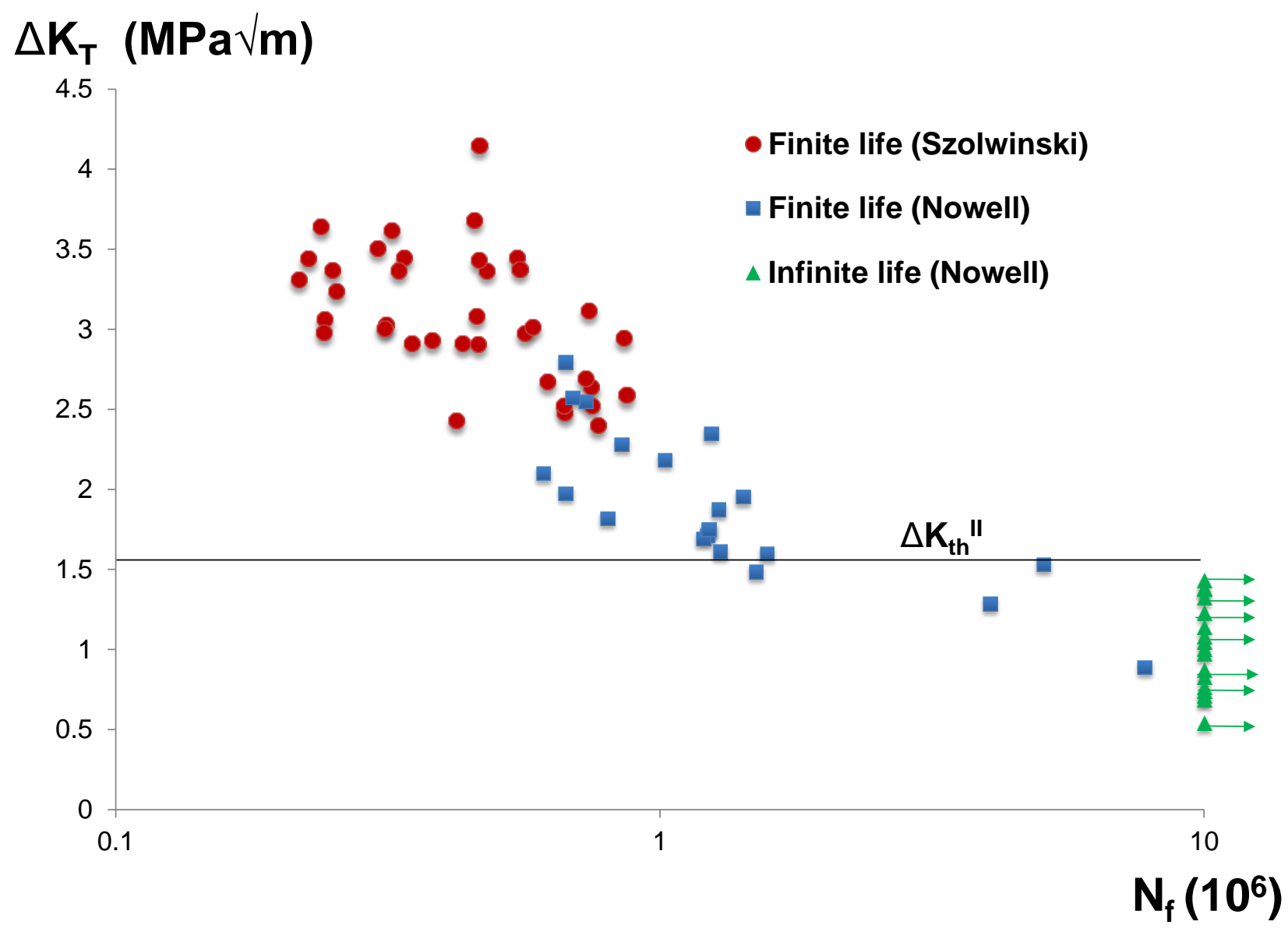

Figure 4. Total fatigue life measured as a function of the range of shear stress intensity $\Delta K_{T}$, for $4 \%$ copper age hardenable aluminium (see text for references). Note the threshold for infinite life. 


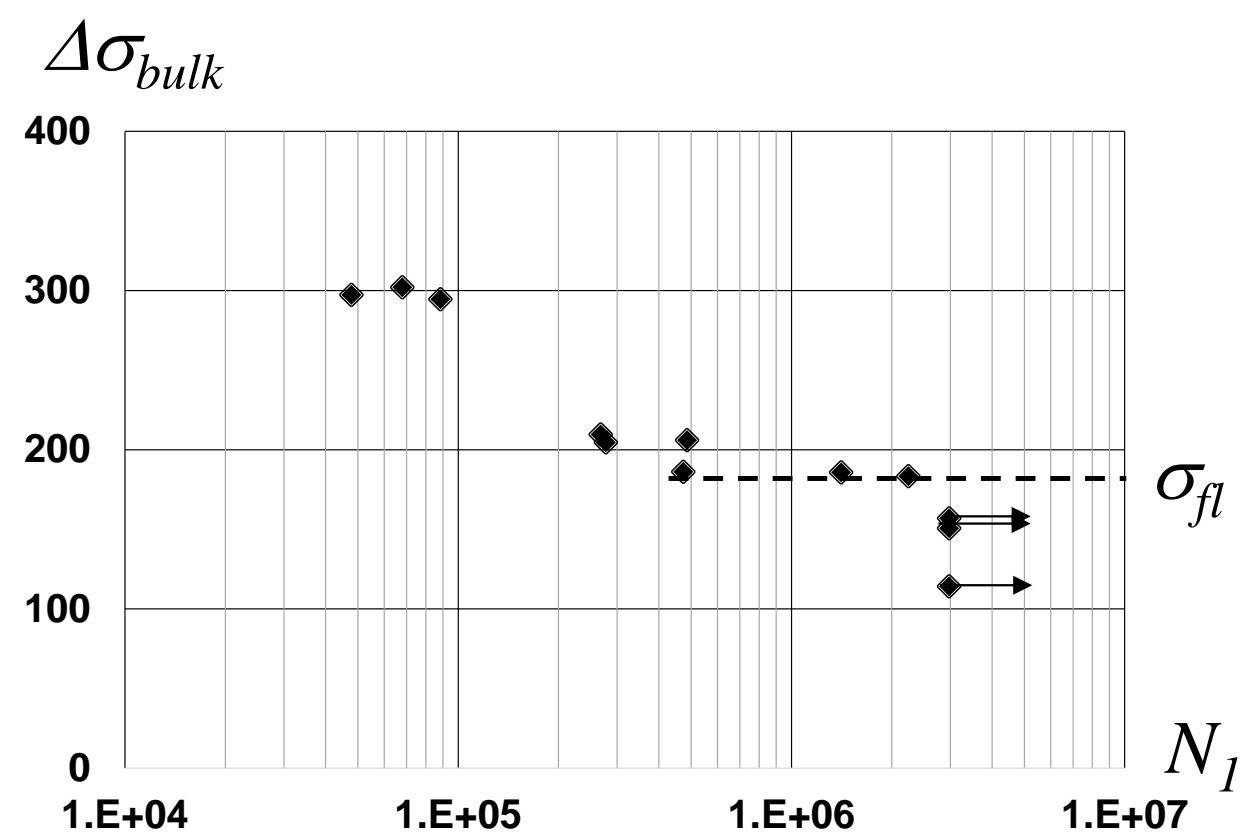

Figure 5. Results of Lehtovaara's fatigue tests using a complete cantilever [17] plotted on conventional S-N axes. The value of S ignores the stress intensification at the cantilever root.

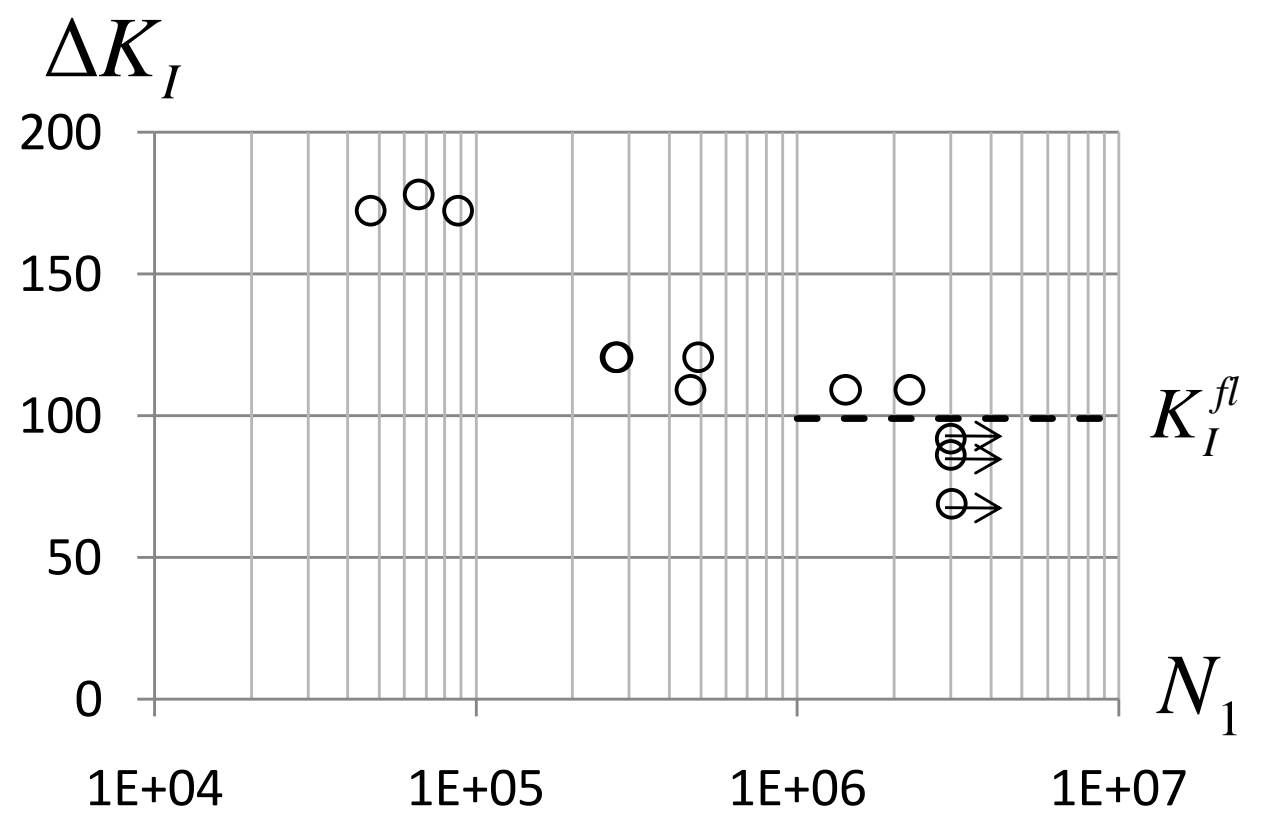

Figure 6. Results shown in Figure 5 but re-plotted in terms of the stress intensity present at the cantilever root and therefore displaying a material property transferrable to other geometries. 